\title{
A five year study of lung lesions in medico-legal autopsies from a pathologist's perspective
}

\author{
Archana Shetty ${ }^{1}$, Vijaya C.,** \\ ${ }^{1}$ Associate Professor, ${ }^{2}$ Professor \& HOD, Dept. of Pathology, Sapthagiri Institute of Medical Sciences \& Research Centre, \\ Bengaluru, Karnataka, India
}

*Corresponding Author:

Email: vijayakrshn0@gmail.com

\begin{abstract}
Introduction: A wide variety of pathologic conditions involve the lungs. The lungs are also involved in almost all terminal events of cardiovascular disease. Autopsy is important not only to determine the state of lungs at death but also to study the morphology of various diseases. Aims and objectives of study are to identify the histopathological spectrum of lung lesions and to find out the frequency of various lung pathologies in respect to age and sex.

Materials and Methods: This was a retrospective study of medico legal autopsies over a period of five years. The lung tissue was fixed and processed. Routine paraffin sectioning was done followed by Haematoxylin and eosin (H and E) staining. Special stains were done when required. Relevant clinical, postmortem findings, gross and microscopic examination findings were recorded.

Results: After thorough histopathological examinations, of total 412 cases, various pulmonary lesions were identified in 349 $(84.6 \%)$ cases, in $23(5.6 \%)$ of cases tissue was autolysed and 40 (9.8\%) of cases were histologically unremarkable. Most commonly affected age group was 30-49 years, with males being predominantly affected. The changes seen were - Congestion \& edema $116(33.2 \%)$, pneumonia 68(19.6\%), interstitial changes $52(15.1 \%)$, emphysema- $26(7.5 \%)$, chronic venous congestion $30(8.6 \%)$, abscess $(1.1 \%)$, haemorrhage $(6.4 \%)$ tuberculosis $-11(3.3 \%)$, bronchiolitis- $3(0.8 \%)$, asthama- $2(0.5 \%)$, ARDS- 7 $(1.9 \%)$, lung cysts- $2(0.5 \%)$

Conclusions: Though the pulmonary diseases are commonly encountered clinically, studying of lung tissues submitted for autopsy, not only gives us an insight into the histology of varying stages of different diseases but also is an important educative tool for the forensic pathologists.
\end{abstract}

Keywords: Edema and congestion, Pneumonia, Tuberculosis, Autopsy, Histopathology.

\section{Introduction}

Lung disorders till date have continued to capture the interest of the medical fraternity because of its varied and complex presentations. Despite availability of modern advanced diagnostic methods, diagnosis is often challenging task for clinicians. Sometimes rapid progression of the disease leaves lesser time for thorough diagnostic workup and invasive procedures. Also, lungs being organs not easily subjected to biopsy early histopathological features of many of the well documented disorders still remains a mystery As a result, histopathological examination of lung autopsy is of great value not only to diagnose the respiratory cause of death if any but also because it enriches our knowledge about lung histology in health and disease. ${ }^{1}$ Studies have documented $20-30 \%$ of sudden deaths being attributed to underlying pulmonary pathology. ${ }^{2}$ Sindu Also, autopsies are great tools of learning for the pathologists as it helps to study the histomorphological course of various infrequent lesions.The lungs are vulnerable for a wide range of inflammatory, infectious, neoplastic and other pathologic conditions and almost always involved secondarily by terminal events of cardiovascular disease. ${ }^{3}$

In addition to ascertain clinico-pathological differences, autopsy has a role in the development of new understanding of old diseases and facilitate the opportunity to uncover the pathophysiology of new diseases. ${ }^{4}$

\section{Materials and Methods}

This was a retrospective cross sectional study was carried out in the Department of Pathology of a medical college attached to a tertiary care hospital, Bangalore over a period of six years from August 2013 to August 2018. Cases that were subjected to medico legal autopsy with lung tissues being submitted for histopathological examination, under the department of forensic medicine during the mentioned period, irrespective of age and sex, were included in the study.

Cases in which the lung tissues were not submitted, cases in which the lung tissue was autolysed and cases in which the microscopic histology was normal were excluded from the study.

Pieces of lungs were received in $10 \%$ formalin from the forensic department of our institute after conducting the medico legal autopsy. Gross examination was made of all the bits, correlating with the clinical findings as mentioned in the request form. These pieces were examined for consistency, colour, and texture, areas of consolidation, infarction, edema, bullae, exuding froth / pus and haemorrhage. Representative bits of 4 to $5 \mathrm{~mm}$ thickness from lung tissues were put in cassettes and subjected to routine 
processing. Paraffin embedding was done and blocks were prepared. All the histological sections were stained with Haematoxylin \& Eosin stain and mounted. Special stains like Zeil Neilson and PAS were asked for when required. The stained sections were then observed under microscope and findings recorded.

\section{Results}

A total of 412 cases were subjected to medicolegal autopsy during the mentioned period of study along with relevant clinical details and autopsy findings being documented .In all cases a minimum of four bits of lung tissue (two from each lung) were sent for examination in ten percent formalin each with an average measurement of $6 \times 4 \times 3 \mathrm{cms}$.

Of the total cases in 23 cases (5.6\%) the lung tissue was autolysed and in another $40(9.8 \%)$ cases the gross and microscopic histopathology was unremarkable and hence were excluded from the study as mentioned above in the criteria. Significant pathological microscopic findings were found in a total of 349 cases (84.6\%). Table 1

Table 1: Distribution of findings in the lung tissue received for histopathological examniation

\begin{tabular}{|l|c|c|}
\hline Overall state of lungs & No. of cases & Percentage \\
\hline Autolysed & 23 & 5.6 \\
\hline Pathological findings & 349 & 84.6 \\
\hline Normal histology & 40 & 9.8 \\
\hline Total & 412 & 100 \\
\hline
\end{tabular}

Out of the 349 cases included in the study, 240 of the cases were those of males, and 109 cases were females, accounting to a male: female ratio of 2.2: 1 . The highest cases received were in the age group of 31- 45 years, with the least cases being from the age group of over $\mathbf{7 5}$ years. Gross examination of the lungs showed the features as given in table 2 .

Table 2: Gross findings of the lungs

\begin{tabular}{|l|l|l|}
\hline Gross feature of lungs & No. of cases & Percentage \\
\hline Haemorrhagic & 47 & 12.8 \\
\hline Consolidated & 152 & 43.6 \\
\hline Focal grey white to brown areas & 32 & 9.8 \\
\hline Unremarkable & 118 & 33.8 \\
\hline Total & 349 & 100 \\
\hline
\end{tabular}

The various histopathological findings that were observed were as follows - edema and congestion, chronic venous congestion, emphysematous changes, pneumonia and haemorrhage in a majority of cases. Also a few cases of Adult Respiratory distress syndrome (ARDS), bronchiolitis, asthma, Tuberculosis, benign lung cysts and granulomatous inflammation were noted. The distribution of the same is given age wise in Table 3 .

Table 3: Age wise distribution of the pulmonary lesions

\begin{tabular}{|l|c|c|c|c|c|c|c|c|}
\hline Pulmonary lesion & $\mathbf{N B}^{\#-15 y}$ & $\mathbf{1 6 - 3 0 y}$ & $\mathbf{3 - 4 5 y}$ & $\mathbf{4 6 - 6 0 y}$ & $\mathbf{6 1 - 7 5 y}$ & $\mathbf{> 7 5 y}$ & $\begin{array}{c}\text { Total } \\
\text { cases }\end{array}$ & Percentage \\
\hline Congestion and edema & 8 & 18 & 46 & 28 & 12 & 4 & 116 & 33.2 \\
\hline $\begin{array}{l}\text { Chronic venous } \\
\text { congestion (CVC) }\end{array}$ & 2 & 5 & 11 & 6 & 4 & 2 & 30 & 8.6 \\
\hline Pneumonia & 7 & 11 & 20 & 21 & 8 & 1 & 68 & 19.6 \\
\hline Interstitial changes & 5 & 9 & 12 & 16 & 8 & 2 & 52 & 15.1 \\
\hline Emphysematous changes & 3 & 7 & 8 & 5 & 2 & 1 & 26 & 7.5 \\
\hline Hemorrhage & 2 & 5 & 6 & 7 & 1 & 1 & 22 & 6.4 \\
\hline Granulomatous lesion & 0 & 2 & 2 & 1 & 1 & 0 & 6 & 1.7 \\
\hline Tuberculosis & 0 & 3 & 6 & 2 & 0 & 0 & 11 & 3.3 \\
\hline Abscess & 0 & 0 & 1 & 1 & 1 & 1 & 4 & 1.1 \\
\hline Bronchiolitis & 0 & 0 & 1 & 1 & 1 & 0 & 3 & 0.8 \\
\hline ARDS & 2 & 0 & 2 & 1 & 1 & 1 & 7 & 1.9 \\
\hline Asthma & 0 & 0 & 1 & 1 & 0 & 0 & 2 & 0.5 \\
\hline Benign cysts & 0 & 0 & 1 & 1 & 0 & 0 & 2 & 0.5 \\
\hline
\end{tabular}

NB- New born $\mathrm{y}=$ age in years 
Congestion and edema was the most common pathological findings accounting for $33.2 \%$ of the cases. The second most commonly observed finding was Pneumonia (lobar pneumonia - 48 cases, interstitial pneumonia -12 cases and bronchopneumonia 8 cases). A note was also made on the stages of lobar pneumonia seen in all the cases, the distribution of which among the sexes is shown in table 3. Red hepatisation was the most commonly observed stage in both males and females as seen in table 3. Only few cases showing the fibrosis / signs of resolution were seen.

Table 4: Distribution of cases according to stages in lobar pneumonia

\begin{tabular}{|l|c|c|c|}
\hline \multicolumn{1}{|c|}{ Stage of Pneumonia } & Males & Females & Total \\
\hline Congestion & $2(4.1 \%)$ & $1(2.1 \%)$ & $3(6.2 \%)$ \\
\hline Red Hepatization & $7(14.6 \%)$ & $3(6.2 \%)$ & $10(20.8 \%)$ \\
\hline Grey Hepatization & $13(27.1 \%)$ & $5(10.4 \%)$ & $18(37.5 \%)$ \\
\hline Resolution & $4(8.3 \%)$ & $3(6.2 \%)$ & $7(14.5 \%)$ \\
\hline & $26(54.1 \%)$ & $12(45.9 \%)$ & $48(100 \%)$ \\
\hline
\end{tabular}

The distribution of lesions among males and females were as given in table 4

Table 5: Sex wise distribution of pulmonary lesions

\begin{tabular}{|l|c|c|c|c|}
\hline Pulmonary lesion & Males & Females & Total cases & Percentage \\
\hline Congestion and edema & $82(23.5 \%)$ & $34(9.7 \%)$ & 116 & 33.2 \\
\hline Chronic venous congestion & $19(5.4 \%)$ & $11(3.1 \%)$ & 30 & 8.6 \\
\hline Pneumonia & $42(12.1 \%)$ & $26(7.5 \%)$ & 68 & 19.6 \\
\hline Interstitial changes & $24(6.9 \%)$ & $28(8.2 \%)$ & 52 & 15.1 \\
\hline Emphysematous changes & $19(5.5 \%)$ & $7(2.5 \%)$ & 26 & 7.5 \\
\hline Hemorrhage & $15(4.3 \%)$ & $7(2.1 \%)$ & 22 & 6.4 \\
\hline Granulomatous lesion & $5(1.4 \%)$ & $1(0.3 \%)$ & 6 & 1.7 \\
\hline Tuberculosis & $9(2.7 \%)$ & $2(0.6 \%)$ & 11 & 3.3 \\
\hline Abscess & $2(0.5 \%)$ & $2(0.5 \%)$ & 4 & 1.1 \\
\hline Bronchiolitis & $2(0.5 \%)$ & $1(0.3 \%)$ & 3 & 0.8 \\
\hline ARDS & $4(1.1 \%)$ & $3(0.8 \%)$ & 7 & 1.9 \\
\hline Asthma & $1(0.25 \%)$ & $1(0.25 \%)$ & 2 & 0.5 \\
\hline Benign cysts & $2(0.5 \%)$ & $0(0 \%)$ & 2 & 0.5 \\
\hline Total Cases & $240(68.7 \%)$ & $109(31.4 \%)$ & 349 & $100 \%$ \\
\hline
\end{tabular}

Interstitial changes like fibrosis, non specific inflammation were seen in $15.1 \%$ of the cases. Haemorrhage both intra - alveolar and interstitial were seen in a total of 22 cases. One case showed the presence of micro - thrombi in the blood vessels. Abscess - comprising of dense acute inflammatory infiltrate of neutrophils to the extent of obscuring the lung parenchymal details was seen in four cases, distributed equally among males and females. Emphysematous changes showing dilated and thinned out alveoli were seen in $7.5 \%$ of the cases.

Granulomatous lesions were seen, with 4 lesions showing foreign body giant cell reaction. However, the two other lesions were not indicative any specific aetiology. 11 cases of Tuberculosis were seen during the study period, out of which two were cases of disseminated tuberculosis with lesions involving other organs, The other 9 cases were of pulmonary tuberculosis, one was a case of tuberculosis with superadded pneumonia. Classic caseating epitheloid cell granulomas with Langhan's giant cells were seen in all cases. Two of the total eleven cases of TB were also HIV positive as per given details.
A single case of ARDS with classical findings were reported. Also reported were two benign solitary lung cysts.

Figures of a few cases with classical findings are as given below. 


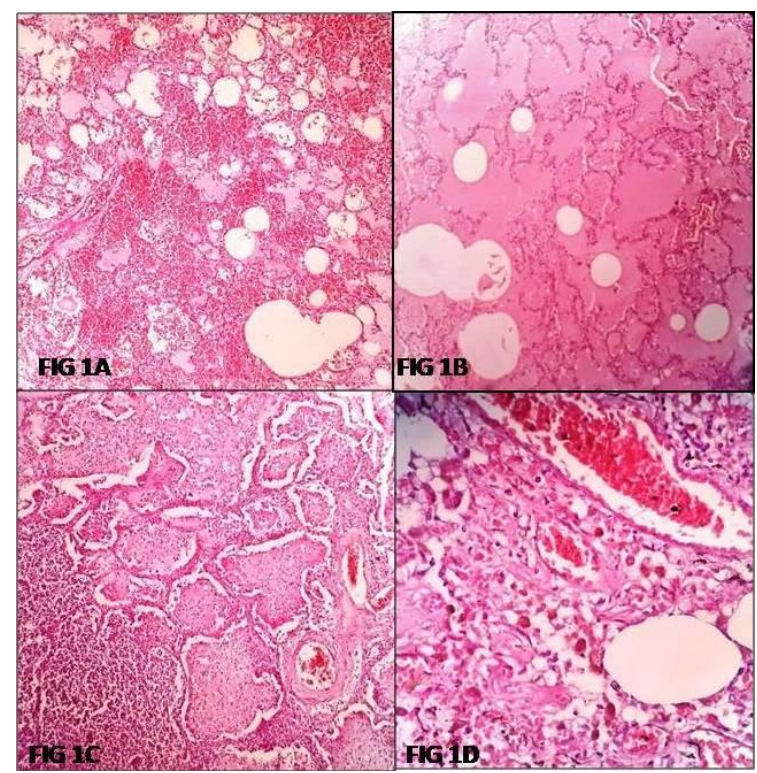

Fig. 1: Intra alveolar and parenchymal haemorrhage; 1B :Pulmonary Edema; 1C: Grey Hepatization stage of lobar pneumonia; 1D : CVC Lung

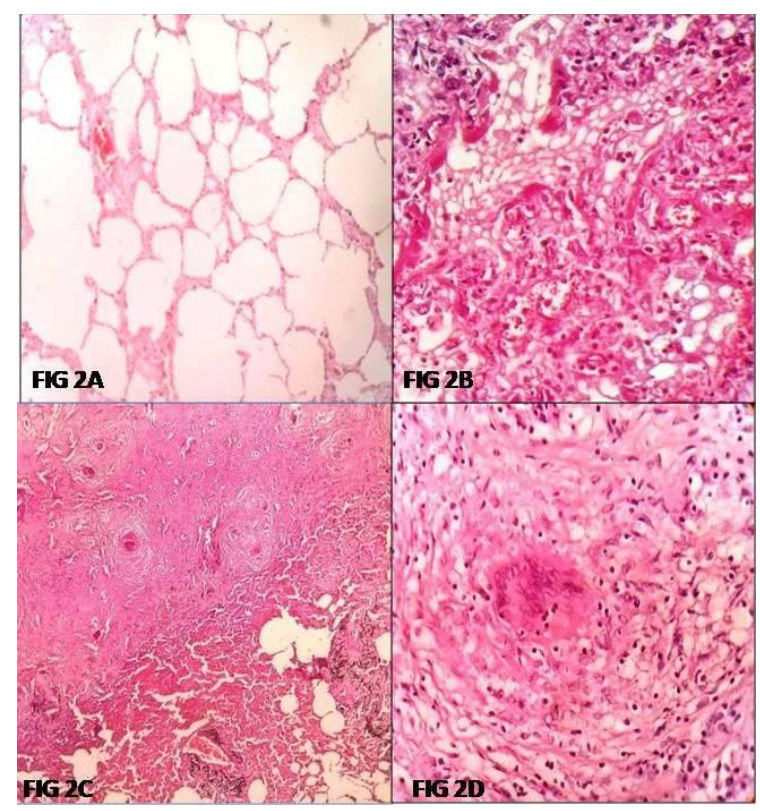

Fig. 2A: Emphysematous lungs; 2B: Diffuse alveolar damage showing hyaline membranes; 2C: Caseating granulomas in lung parenchyma; 2D: Epithelioid cell granuloma with Langhan's giant cells

\section{Discussion}

The lungs are vital organs that are frequently involved in various kinds of benign and neoplastic lesions commonly encountered in clinical practice. The clinical and radiological findings in pulmonary diseases are nonspecific and often ancillary tests are relied upon for specific diagnoses, which are necessary not only to prevent the rapid progression of the disease but also to avoid patients from being subjected to more invasive procedures, especially in a developing country like ours. ${ }^{5}$ However due to the difficulty in accessibility of this organ to be subjected to biopsy / FNAC histopathological findings in various conditions at varying stages are challenging to study in live patients. It is the autopsy studies that contribute a great knowledge to our understanding of the morphology of the pulmonary tissue in various pulmonary and extra pulmonary diseases. ${ }^{6}$

It also helps us to determine the leading causes of death enabling timely preventive measures, which is the least expensive strategy for preventing pulmonary dysfunction. ${ }^{7}$

In the present study the maximum cases of pulmonary lesions was seen in the third to fouth decade of life. This is comparable to the studies by Patel $\mathrm{C}^{3}$ Selvambigai $\mathrm{G}^{8}$ and Tahir et al. ${ }^{9}$.Males were more commonly affected than females, in accordance with studies by Kunawar et al[4] ,Bal et al ${ }^{10}$ and Selvam et al. ${ }^{11}$

Congestion and edema, two events attributed to cardiac dysfunctioning were the most common findings in our study $(33.2 \%)$, similar to study by Kurawar et $\mathrm{al}^{4}$ and 6 Chauhan et al. ${ }^{6}$ Immediate mortality in trauma patients is usually attributed to post-trauma complications like severe organ injury and shock. The lung being the only organ to get affected by the stressed left ventricle, the finding of congestion and edema justifies the failure of the ventricles to sustain the various pathophysiologies leading to the death.

Infectious diseases till date continue to be a major contributor to the mortality and morbidity on our country. In the present study, second most common finding was $19.6 \%$ of lobar pneumonia. Interstitial pneumonia and bronchopneumonia accounted for only few countable cases. Hospital-acquired infections are the predominant contributors of fatality in patients surviving after the initial few days of trauma. Majority of trauma patients on ventilators develop pneumonia if the ventilators are used for more than 5 days. Ventilator-associated pneumonia (VAP) is reported to contribute to a significant proportion of mortality in trauma patients Since trauma victims are predominantly middle aged, otherwise healthy males, pneumonic infections in these patients may truly represent a hospital acquisition. ${ }^{12}$ The stages of pneumonia seen as categorized in our study explain the time evolved in various cases from the time of insult till death.

Tuberculosis (TB) till date continues to be one of the most pressing disease in a developing country like ours. ${ }^{13}$ Due to the stigma associated with this disease often there is a delay in diagnosis and treatment. An estimated 1.96 million cases are reported annually accounting for one-fifth of the global incidence of TB. ${ }^{14}$ In a study by Kurawar et $\mathrm{al}^{4}$ tuberculosis was seen in 32 $(2.53 \%)$ cases out of 1263 cases studied, of which, majority - $22(1.74 \%)$ cases were males and the commonest age group was 40-49 years. Our study is in 
agreement with the above mentioned Indian studies, with having documented a $3.3 \%$ of cases of tuberculosis highest cases being in the $31-45$ years age group. One case had presented with Tuberculosis pneumonia. Tahir et $\mathrm{al}^{9}$ in their study found $19 \%$ cases of tuberculosis with male preponderance and most of them being below 50 years of age . Aziz et al. ${ }^{15}$ In their study on 46 cases of tuberculosis of lung observed a bimodal distribution with a younger age group of less than 30 years and an older age group of more than 50 years To date, autopsy studies have found the presence of Mycobacterium tuberculosis infection in 5-59\% of cases. One of the most common characteristics of tuberculosis is its chronic form of development and progression. In a developing country like ours, where rural population contribute a major share, many times symptoms of Tuberculosis are not taken to the treatment level.

Flavin et $\mathrm{al}^{16}$ reported 15 cases $(0.3 \%)$ of active tuberculosis from 4930 autopsies over a period of 14 years, out of which $67 \%$ remained undiagnosed till autopsy. ${ }^{17}$ Forty five cases $(61.64 \%)$ were specifically assigned to tuberculosis $(16.42 \%$ of total sudden deaths). A steady rise in number of sudden deaths due to tuberculosis was observed during the study period. In our study most of the patients were young, in the third decade of life with male predominance, with Tuberculosis not being the primary cause of death.

A few differential diagnoses must be thought of during the reporting of pulmonary granulomas. Nonneoplastic granulomatous diseases like Wegener's granulomatosis and Churg-Strauss syndrome on histomorphology show necrotizing granulomas with features of vasculitis. Granulomas of tuberculosis may also involve blood vessels resembling a true primary vasculitic disorder posing a diagnostic challenge Many infectious granulomatous lesions may also mimic tuberculosis. Fungal infections especially histoplasma show necrosis similar to tuberculosis. Special stains come to rescue by identifying fungal elements if any in such cases. ${ }^{18}$ Though Zeil Neilson staining strongly identifies the TB bacillus, Acid fast bacilli (AFB) positivity in smears and histological specimens depends on the bacillary load of the specimen and the type of the tissue submitted

Tuberculosis when associated with HIV infection is aggressive and is characterized by widespread dissemination. ${ }^{14}$ Tuberculosis was the most common finding at autopsy with $78 / 125$ cases $(62.4 \%)$, of which 66/78 (84.6\%) were HIV-infected. ${ }^{19}$

Despite the use of prophylactic antibiotics over the course of infection, the lungs are the organs most frequently affected by HIV/AIDS. As a result failure of the respiratory system is one of the main causes of death in HIV/AIDS patients..$^{20}$ Little is known about the causes of death or the histological pulmonary findings in patients with HIV/AIDS at autopsy. Alveolar Interstitial Pneumonia (AIP) and Diffuse Alveolar
Damage (DAD) have been documented to be the most common findings in the diseased lungs in a first of its kind study done by Soiro $\mathrm{Am}$ at $\mathrm{al}^{20}$ who studied autopsy findings in HIV / AIDS deaths. In our study two cases which were HIV positive had features of interstitial pneumonia and Tuberculosis attributed to the immune compromised status of the infected persons.

Emphysema is destruction of alveolar septal walls. Decades ago studies have shown the prevalence of emphysema to be three times more in males than in males. However a study by Marel et al had shown that emphysema detected during life was more frequent in females than in males (43 vs. 40 in their study, whereas at autopsy, this condition was more frequently identified in males than females. Kare et $\mathrm{al}^{1}$ and Chauhan et $\mathrm{al}^{6}$ in their study have noted the percentage of emphpysematous cases to be $8.2 \%$ and $7.76 \%$ respectively. Emphysematous lesions are frequently combined with inetstitial fibrosis, indicating a poor prognosis. $^{21}$ In our study we found $7.5 \%$ of emphysematous cases being seen predominantly in males, similar to the latter studies.

ARDS or diffuse alveolar damage disease as seen in histology is a complex inflammatory condition involving the alveolo- capillary barrier ${ }^{22}$ was seen only in $1.9 \%$ of cases in our study, similar to other studies on lung autopsies. ${ }^{2,5}$ Histological criteria for ARDS is defined as the presence of hyaline membranes and at least one of the following findings: necrosis of type I pneumocytes or endothelial cells, edema, organizing interstitial fibrosis and prominent proliferation of type II pneumocytes which were seen the present study also.

A very few cases of benign solitary cysts, bronchitis and lung abscesses were also seen.

\section{Conclusion}

The present study documents the histomorphological spectrum of lung lesions seen in autopsies. Though the pulmonary diseases are commonly encountered clinically, studying of lung tissues not only gives us an insight into the histology of varying stages of different diseases but also is an important educative aid to the forensic pathologists. It also highlights the importance of identifying potentially lethal infections like Tuberculosis in developing countries as it can be a hidden contributor to moratality and morbidity.

\section{Acknowledgement}

We would like to thank the all staff of the

Department Of Forensic Medicine of our Institute for their valuable support for this study.

Funding: No funding sources.

Conflict of interest: None declared. 


\section{References}

1. Khare P, Gupta R, Ahuja M, Khare N, Agarwal S, Bansal D. Prevalence of Lung Lesions at Autopsy: A Histopathological Study. J Clin Diagnostic Res 2017;11(5):EC13-EC16. doi:10.7860/JCDR/2017/24747.9827.

2. Sindu V, Dhanalakshmi A. Histopathological Analysis of Lung In Sudden Natural Death. IOSR J Dent Med Sci 2016;15:(10):37-42.

3. Patel S, Rajalakshmi BR, Manjunath GV. Histopathologic Findings in Autopsies with Emphasis on Interesting and Incidental Findings-A Pathologist's Perspective. J Clin Diagnostic Res 2016;10(11):EC08EC12

4. Kurawar Rr and Vasaikar MS. Spectrum of histomorphological changes in lungs. Ann Pathol Laboratory Med 2017;04(1):106-12.

5. Kasper, Fauci, Hauser, Longo, Jameson, Loscalzo. Harrison's principles of internal medicine, 19th ed. Vol 2, Mc Graw Hill; Indian edition:2015.

6. Chauhan G, Agrawal M, Thakkar N, Parghi B. Spectrum of histopathological lesions in lung autopsy. J Res Med Den Sci 2015;3(2):109-12.

7. Ghosal R, Kloer P, Lewis KE. A review of novel biological tools used in screening for the early detection of lung cancer. Postgraduate Med J 2009;85:358-63.

8. Selvambigai G, Amudhavalli S, Chakravarthi DCD, Ravi S. Histopathological study of lung in autopsy cases: a prospective study. Int J Res Med Sci 2016;4:4816-19.

9. Tahir TM, Rehmna F, Anwar S, Kamal F. Pattern of pulmonary morphological lesions seen at autopsy. Biomedica 2013;29:64-8.

10. Bal Manjeet S, Sethi PS, Suri Anil K, Bodal Vijay K, Kaur G. Histopathological pattern in lung autopsies JPAFMAT 2008;8(2):29-31.

11. Selvam V, Thamil Selvi R, Subramnium PM, Vijaynath V. Prevalence of common diseases in lungs and liver: A histopathological study. J Pharmaceut Biomed Sci 2011;12(12):1-5.

12. Lalwani S, Mathur P, Tak V, Janani S, Kumar SI, Bagla $\mathrm{R}$, et al. Diagnosis of ventilator-associated pneumonia: comparison between ante-mortem and post-mortem cultures in trauma patients. Indian J Med Microbiol 2014;32:294-300.

13. Sangma MM, Devi TM, Sarangthem B, Keisham S, Devi PM. Prevalence of tuberculosis: A study in forensic autopsies. J Med Soc 2014;28:162-5.

14. Gupta M, Lobo F D, Adiga D S A et al. A Histomorphological Pattern Analysis of Pulmonary Tuberculosis in Lung Autopsy and Surgically Resected Specimens. Pathol Res Int J 2016;8132741

15. Aziz R, Khan AR, Qayum I, Mannan M, Khan MT and Khan N. Presentation of pulmonary tuberculosis at Ayub Teaching Hospital Abbottabad. J Ayub Med College, Abbottabad 2002;14(1):6-9.

16. Flavin R. J., Gibbons N., O'Briain D. S. Mycobacterium tuberculosis at autopsy - exposure and protection: an old adversary revisited. J Clin Pathol 2007;60(5):487-91.

17. Rastogi P, Kanchan T, Menezes RG. Sudden unexpected deaths due to tuberculosis: An autopsy based study. $J$ Foren Med Toxicol 2011;28:81-3.

18. Mukhopadhyay S and Gal AA. Granulomatous lung disease an approach to the differential diagnosis. Arch Pathol Laboratory Med 2010;134(5):667-90.

19. Bates M, Mudenda V, Shibemba A, Kaluwaji J, Tembo J, Kabwe $M$ et al. Burden of tuberculosis at post mortem in inpatients at a tertiary referral centre in sub-Saharan Africa: a prospective descriptive autopsy study. Lancet Infect Dis 2015;15:544-51.

20. Soeiro Ade M, Hovnanian AL, Parra ER, Canzian M, Capelozzi VL. Post-mortem histological pulmonary analysis in patients with HIV/AIDS Clinics (Sao Paulo). 2008;63(4):497-502

21. Inomata M, Ikushima S, Awano N, Kondoh K, Satake K, Masuo $\mathrm{M}$ et al. An autopsy study of combined pulmonary fibrosis and emphysema: correlations among clinical, radiological, and pathological features. BMC Pulm Med 2014; 14:104.

22. Pinheiro BV, Muraoka FS, Assis RV, Lamin R, Pinto SP, Ribeiro PJ et al. Accuracy of clinical diagnosis of acute respiratory distress syndrome in comparison with autopsy findings. J Bras Pneumol 2007;33:423-8.

How to cite this article: Shetty A. C. Vijaya. A five year study of lung lesions in medico-legal autopsies from a pathologist's perspective. J Diagn Pathol Oncol 2018;3(4):344-49. 